\title{
Study of different Trends and Techniques in Face Recognition
}

\author{
Divyakant T. Meva \\ Research Scholar \\ Saurashtra University \\ Rajkot, India
}

\author{
C K Kumbharana, Ph.D \\ Associate Professor \\ Saurashtra University \\ Rajkot, India
}

\begin{abstract}
History of Face recognition is old enough to be mature. In 1960s, face recognition became semi-automated. In 1970s, face recognition took another step in automation. In 1988, first semi-automated facial recognition system was deployed. In 2001, automated face recognition captured attention of public at SuperBowl event to capture surveillance images. Now a day, every country in the world is using this technology for different purposes. In this paper, we have discussed some novel techniques and algorithms for face recognition of the current trends.
\end{abstract}

\section{General Terms}

Face recognition, Biometrics

\section{Keyword}

Face Recognition, holistic approach, feature based approach, hybrid methods, PCA, LDA, LFA, FDA, HMM

\section{INTRODUCTION}

Now a day, security issues have achieved a lot of attention of IT industry. Specially, various biometric techniques are playing an important role in this matter. Whether it is about managing security at airports or at border of the country, biometrics is the powerful solution of security problems. Face recognition methods can be implemented in two different ways: Identification and verification. Application of biometrics at Airports used for surveillance is an example of identification. While applying biometrics at border of the country is an example of verification. Face recognition is used for both the purposes. Face recognition is a mature technology, so there is no doubt about accuracy and applicability of face recognition.

Other applications of face recognition technology are access control, criminal identification, surveillance in cities, human computer interaction (HCI), law and enforcement, smart cards and multimedia management.

The main advantage of face recognition is that it is one of the oldest biometric technologies and is cheaper with compare to other technologies. Though face cannot be considered as a unique feature like finger and iris or retina, but still is having acceptable accuracy rate. Now a day, in the trend of multibiometrics, face recognition can be clubbed together with fingerprint for better solution to minimize chances of false accept or false reject.

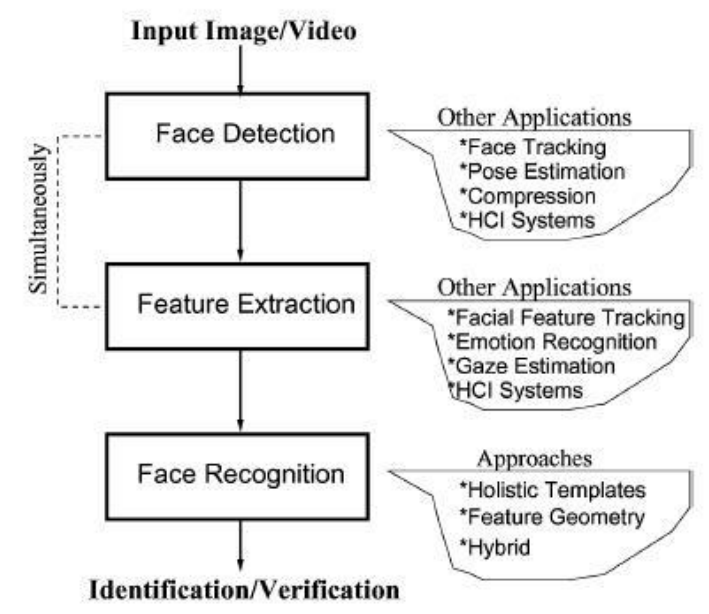

Fig. 1 - Generic face recognition system [1]

A Typical flow of face recognition system is given in Figure 1. Input is taken from either image or video. The first stage in the process is face detection. Here segmentation of face areas from background is done. Face alignment as a part can be applied to achieve more localization and normalization of faces. Face detection also provides estimates of location and scale of each face.

Once face is normalized, feature extraction is performed to get effective information to distinguish faces of different persons. In the last stage of face recognition, feature vector is compared with database of enrolled faces. At the end output comes in term of identification or verification of a person.

\section{DIFFERENT FACE RECOGNITION TECHNIQIES}

As it has been mentioned in abstract, semi automated face recognition came into know in 1988. But since last 20 years, the work is going on for automated face recognition system and enhancements in those systems. In the survey of face recognition techniques given by Zhao et al. in 2003 [1], they categorized face recognition systems into two categories:

1. Appearance based - this technique uses holistic texture features and they are applied to either whole face or specific regions of the face image.

2. Feature based - this technique uses geometric facial features like mouth, eyes, cheeks etc. and geometric relationship between these features.

3. Hybrid methods - As human being, we are used to for matching face as a whole with holistic approach as well as with the help of features of the faces. 
Similar approach can be used for machine based face recognition also.

They suggested that compared to appearance based approaches, feature based approaches are less sensitive to changes in illumination and viewpoint. But feature extraction techniques for these approaches are still not reliable and accurate as expected.

\subsection{Appearance based (holistic) approach}

In this approach, one of the most widely used methods is algorithms or techniques based on PCA (Principal Component Analysis). The first successful experiment of face recognition with machine was made by Turk and Pentland in 1991 [2] with Eigenfaces. In this method, every face of the database is represented as a vector of weights; these weights are obtained by projecting the image into Eigenface components with simple inner product operation. The test image for identification is represented by its vector of weights and then compared with database and locates the sample with closest weights compared to test image.

Moghaddam and Pentland in 1997 [3] extended this method to Bayesian approach. But the distribution of probability among the sample data sets was a problem with this method. This problem was solved with Multiclass problem by using similarity measure based on Bayesian Analysis.

Face recognition system using Linear Discriminant Analysis (LDA) has been successful. LDA training is performed with Scatter Matrix Analysis.

Belhumeur et al. in 1997 [4] compared performance of four different methods. They compared (1) a correlation-based method, (2) a variant of the linear subspace method (3) an Eigenface method, and (4) a Fisherface method. The results showed that Fisherface method performs better than other three methods.

Liu and Wechsler in 2000 [5] presented an Evolution Pursuit (EP) based representation and its application for face recognition. They identified better performance compared to Eigenfaces and improved generalization capabilities compared to Fisherface.

Another technique was suggested by Lin et al. in 1997 [6]. They proposed system based on Probabilistic Decision Based Neural Network (PDBNN). The unique characteristic was the modular structure of PDBNN.

Appearance based methods are more popular and successful compared to feature based methods.

LDA based methods have performed well compared to PCA based methods. LDA optimizes low dimensional representation of face images. LDA faces the problem of SSS (Small Sample Size). There are several variants of LDA called Liu LDA, Chen LDA, D-LDA and modified Chen LDA to overcome the problem of SSS.

Recent trend adopts frequency domain analysis methods like DCT (Discrete Cosine Transformation), DFT (Discrete Fourier Transformation), and DWT (Discrete Wavelet Transformation) for face recognition. Here images signals are transformed from spatial domain to frequency domain. These methods are data independent [12]. Feature fusion is one of the ways to improve the performance. But it suffers from the problem of high dimensionality of multiple features (combined features).

\subsection{Feature based approach}

The earlier methods of face recognition were working with structural matching. These methods were based on geometry of local features. They were using the features like width of the head, distance between eyes, and the features of mouth, nose and chins [7].

Hidden Markov Model (HMM) based methods use lines of pixels covering eye, nose, mouth, chins etc [8].

The other successful method in this approach is Graph Matching System based on Dynamic Link Architecture (DLA). Another method based on Convolutional Neural Network $(\mathrm{CNN})$ was developed by Lawrence et al. using unsupervised learning method [9].

\subsection{Hybrid methods}

This approach used holistic methods and local features both. Modular Eigenface approach uses global Eigenfaces and local eigenfeatures both [10].

Local Feature Analysis (LFA) improves estimation of higher order eigenmodes. LFA is biologically inspired feature analysis method. Hybrid methods clubs PCA and LFA for better performance of the system.

Another method in this area is Component based detection/ recognition proposed by Heisele et al. in 2001 [11]. Here decomposition of face into a set of facial components like mouth and eyes which are interconnected with some geometry is done.

But the drawback of this system is that it requires large number of images in training set as it is dealing with different poses of the same face due to changes in the head pose.

\section{EXPERIMENTAL RESULTS OF VARIOUS TECHNIQUES}

As shown in the previous section, there are three different approaches for face recognition. Here are some of the experimental results of these methods.

\subsection{Appearance (holistic) based approach}

Liau et al. performed experiments with spatial domain and frequency domain and achieved the following results [12].

Table 1. Spatial domain results

\begin{tabular}{|l|c|}
\hline \multicolumn{1}{|c|}{ Method } & Recognition rate (\%) \\
\hline PCA & 89.5 \\
\hline Chen LDA & 90.5 \\
\hline D-LDA & 89.5 \\
\hline Modified Chen LDA & 91.5 \\
\hline
\end{tabular}

As shown in Table 1, Modified Chen LDA outperforms other methods in spatial domain.

Table 2. Frequency domain result

\begin{tabular}{|l|c|}
\hline \multicolumn{1}{|c|}{ Method } & $\begin{array}{c}\text { Recognition rate } \\
(\boldsymbol{\%})\end{array}$ \\
\hline DCT+ Liu LDA & 94 \\
\hline DCT+ D-LDA & 94 \\
\hline
\end{tabular}




\begin{tabular}{|l|c|}
\hline DFT+ Chen LDA & 94 \\
\hline DFT+ Modified Chen LDA & 96.5 \\
\hline DFT+ D-LDA & 92 \\
\hline DWT+ Chen LDA & 91 \\
\hline DWT+ Modified Chen LDA & 93.5 \\
\hline DWT+ D-LDA & 91.5 \\
\hline
\end{tabular}

They defined 3 parallel models for experiments shown below:

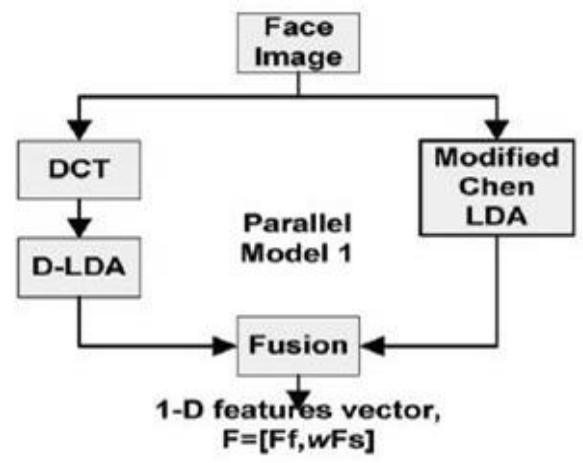

Fig 2: Parallel model - 1

Table 3.Performance in Parallel models

\begin{tabular}{|c|c|}
\hline Method & Recognition rate (\%) \\
\hline Parallel model 1 & 97.5 \\
\hline Parallel model 2 & 99 \\
\hline Parallel model 3 & 96.5 \\
\hline
\end{tabular}

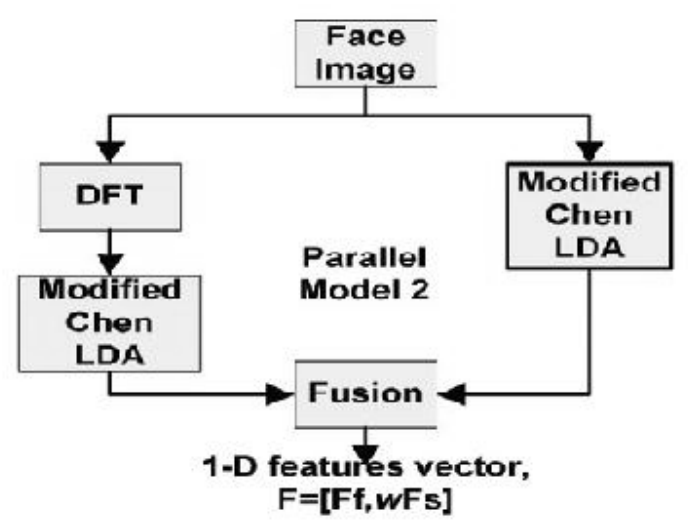

Fig 3: Parallel model - 2

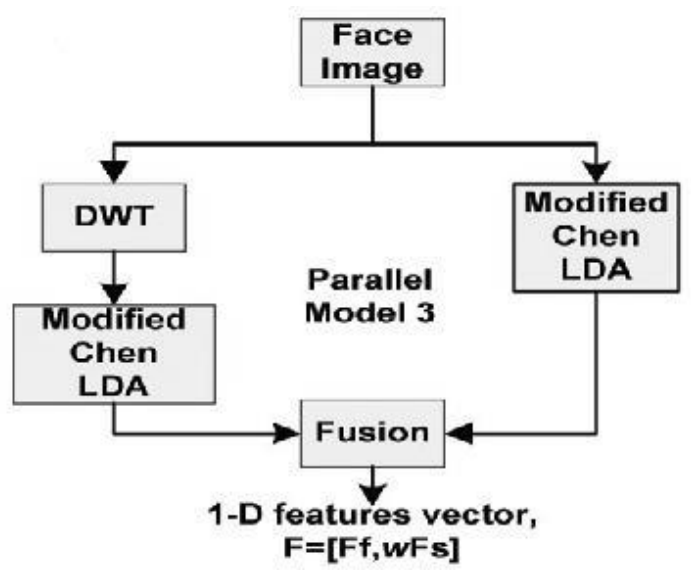

Fig 4: Parallel model - 3

Taranpreet Singh applied PCA based face recognition with neural network back error propagation learning algorithm. He was able to achieve the results with neural network classifier [13] shown in table 4.

Table 4. Performance of PCA based face recognition with neural network back propagation

\begin{tabular}{|c|c|c|c|c|}
\hline $\begin{array}{l}\text { Feature } \\
\text { extractor }\end{array}$ & $\begin{array}{l}\text { FAR } \\
\%\end{array}$ & $\begin{array}{l}\text { FRR } \\
\% \\
\end{array}$ & $\begin{array}{l}\text { HTER } \\
\% \\
\end{array}$ & Scenario \\
\hline \multirow[t]{2}{*}{$\mathrm{PCA}$} & 5.820 & 5.560 & 5.690 & \begin{tabular}{l}
\multicolumn{2}{l}{ Verification } \\
using original \\
image
\end{tabular} \\
\hline & 7.340 & 7.780 & 7.560 & $\begin{array}{l}\text { Verification } \\
\text { using histogram } \\
\text { equalization }\end{array}$ \\
\hline
\end{tabular}

Muhammad Akmal Khan et al. carried out experiments with ORL face database. They tried to improve face recognition rate with the help of Sub Holistic (SH) PCA - (SHPCA) method [14].SH-PCA gave improvement of $6 \%$ correct recognition for complete ORL database. The following table shows performance aspects in terms of correct detection percentage of experiments:

Table 5. Comparison of PCA and SHPCA for correct detection rate

\begin{tabular}{|c|l|l|}
\hline No. of classes & PCA & SHPCA \\
\hline 10 & 95 & 97 \\
\hline 15 & 92 & 96 \\
\hline 20 & 87 & 91 \\
\hline 25 & 88 & 92 \\
\hline 30 & 88 & 91 \\
\hline 35 & 85 & 89 \\
\hline 40 & 82 & 87 \\
\hline
\end{tabular}

\subsection{Feature based approach}

In 1992, Craw et al, applied the concept of local features in face. They used priori information to locate local information. They considered two part implementation: the first was designed to identify individual features like eyes, chin, cheek, hair, mouth, mouth-bits head outline and nose. The second part refined features found in first part using priori information to locate 40 face features. With this method, they achieved $95 \%$ accuracy in their implementation [15].

Manjunath et al. proposed a method which was not considering prior information. They suggested three stage approach for face verification [16]. In the first stage, features were extracted with the use of Gabor wavelet. In the second stage, graph like data structure was created used to represent face features found as a collection of interconnected nodes. They created two graphs: Input face graph - to represent probe face and Model face graph - representing a gallery face. In the third stage, input face graph matched with model face graph to determine identity. They used 306 faces data set. They achieved $86 \%$ recognition rate when probe face was within first rank. $96 \%$ recognition rate was found when probe face was found in first three ranks.

Lades et al. proposed Dynamic Link Architecture which was an extension to Artificial Neural Network (ANN) [17].

Wiskott et al. made extension in Dynamic Link Architecture implementation with multiple jets at each node [18]. They called this as extension Elastic Bunch Graph Matching. They used FERET and Bochum dataset. They found 98\% accuracy with FERET data set. For Bochum data set, they got $94 \%$ accuracy. 


\subsection{Hybrid methods}

Pentlad et al. [10] suggested two approached to handle multiple face views. In the first approach, Eigenface is constructed by using all face views. The second approach suggested by them is View based eigenspaces. Accuracy rate is good in second approach compared to first.

LFA, biologically inspired method for feature analysis is efficient one than PCA. But it is advisable to combine LFA with PCA to have even better performance.

Sarala Ramkumar and Silambarasan Kaliamoorthi proposed hybrid model by combining PCA and FDA (Fisher Discriminant Analysis) to reduce dimensionality [19].Individual persons images were classified into different classes. They used YALE and ORL database and attained 96\% recognition rate with their algorithm implementation.

Rui Huang et al. presented hybrid face recognition method by combining holistic anf feature analysis method. They used Markov Random Field (MRF) model [20]. They performed experiments with several face dataset like YALE, ORL and FERET. Along with MRF model, they tested PCA, LDA and Nearest-Neighbor method.

They took various images of a subject ranging from 1 to 10 . The results of this experiment are shown below:

Table 6. Performance comparison of MRF model with other methods [20].

\begin{tabular}{|l|l|l|l|l|l|}
\hline K & Database & NN & PCA & LDA & MRF \\
\hline 10 & YALE & 83.07 & 81.73 & 100 & 99.33 \\
\hline 9 & ORL & 97.90 & 95.20 & 93.75 & 96.75 \\
\hline 5 & FERET & 89.23 & 79.20 & 88.37 & 99.71 \\
\hline
\end{tabular}

\section{CONCLUSION}

Holistic methods are popular since last few years due to their performance and efficiency. However, they are dependent on careful positioning of the face images into the same canonical pose, which is very tough task. In contradiction, some local feature-based approaches can achieve good recognition performances without additional alignment. But their computational process is heavier than holistic approaches. Hybrid methods provides improved recognition rate and now a day these methods are popular. Hybrid methods are giving consistent performance with all databases. But we cannot forget traditional approached like PCA, LDA or ICA for face recognition as hybrid methods are based on these traditional methods.

\section{REFERENCES}

[1] Zhao W. et al., Face Recognition - A Literature Survey, ACM Computing Surveys, Vol. 35, No. 4, December 2003, pp. 399-458

[2] Turk, M. and Pentland, A., Eigenfaces for recognition, J. Cogn. Neurosci. 3, 1991, 72-86

[3] Moghaddam, B. and Pentlad, A., Probabilistic visual learning for object representation, IEEE Trans. Patt. Anal. Mach. Intell. 19,1997, 696-710.

[4] Belhumeur P N, Hespanha J P and Kreigman D J, Eigenfaces vs. Fisherfaces: Recognition using class specific linear projection. IEEE Trans. Patt. Anal.Mach. Intell. 19, 2007, 711-720.
[5] Liu C, Andwechsler H, Evolutionary pursuit and its application to face recognition, IEEE Trans. Patt. Anal. Mach. Intell. 22, 2000a., 570-582

[6] Lin, S. H., Kung, S. Y., And Lin, L. J., Face recognition/ detection by probabilistic decision based neural network, IEEE Trans. Neural Netw. 8, 1997, 114 132

[7] Kelly, M. D., Visual identification of people by computer, Tech. rep. AI-130, Stanford AI Project, Stanford, CA.

[8] Samaria, F. And Young, S., HMM based architecture for face identification, Image Vis. Comput. 12, 1994, 537583.

[9] Lawrence, S., Giles, C. L., Tsoi, A. C., And Back, A. D., Face recognition: A Convolutional neural-network approach. IEEE Trans. Neural Netw. 8, 1997, 98-113

[10] Pentland, A., Moghaddam, B., Starner, T., View-based And modular eigenspaces for face recognition. In Proceedings, IEEE Conference on Computer Vision and Pattern Recognition, 1994.

[11] Heisele, B., Serre, T., Pontil, M., And Poggio, T. , Component-based face detection. In Proceedings, IEEE Conference on Computer Vision and Pattern Recognition, 2001.

[12] Liau et al., " New parallel Methods of Face Recognition", Advances in Face Recognition, I-Tech, 2008, pp 15-26

[13] Taranpreet Singh Ruprah, "Face Recognition Based on PCA Algorithm", Special Issue of International Journal of Computer Science \& Informatics, ISSN: 2231-5292, Vol.- II, Issue-1, 2 , pp 221 - 225

[14] Muhammad Akmal Khan et al., "Face Recognition using Sub - Holistic PCA", British Journal of Science, September 2011, Vol. 1 (1), pp. 111- 120

[15] Craw, I., Tock, D. \& Bennett, A. Finding Face Features, in 'European Conference on Computer Vision', 1992, pp. 92-96.

[16] Manjunath, B., Chellappa, R. \& von der Malsburg, C. (1992), 'A Feature Based Approach to Face Recognition', IEEE Conference Proceedings on Computer Vision and Pattern Recognition pp. 373-378.

[17] Lades, M., Vorbr"uggen, J., Buhmann, J., Lange, J., von der Malsburg, C.,W“urtz, R. \& Konen,W., 'Distortion invariant object recognition in the dynamic link architecture', IEEE Transactions on Computers 42(3),1993, 300-311.

[18] Wiskott, L., Fellous, J., Kr"uger, N. \& von der Malsburg, C., Face recognition by elastic bunch graph matching, in L.C. Jain et al., ed., 'Intelligent Biometric Techniques in Fingerprint and Face Recognition',1999,CRC Press, chapter 11, pp. 355-396.

[19] Sarala Ramkumar \& Silambarasan Kaliamoorthi, "A Hybrid Approach to Face Recognition under Varying Illumination", IJCSET, April 2011, Vol. 1, Issue 3,113117

[20] Rui Huang et al, “ A Hybrid Face Recognition Method using Markov Random Fields”. 